\title{
High Energy Resolution Monochromated EELS-STEM System
}

Ondrej L. Krivanek ${ }^{1}$, Tracy C. Lovejoy ${ }^{1}$, Neil J. Bacon ${ }^{1}$, George J. Corbin ${ }^{1}$, N. Dellby ${ }^{1}$, P. Hrncirik ${ }^{1}$, Matthew F. Murfitt ${ }^{1}$, Gwyn Skone ${ }^{1}$, Zoltan S. Szilagyi ${ }^{1}$, Philip E. Batson ${ }^{2}$ and R.W. Carpenter ${ }^{3}$

${ }^{1}$ Nion, $11028^{\text {th }}$ St, Kirkland, WA 98033, USA

${ }^{2}$ Departments of Physics/Astronomy and Materials Sci/Eng, Rutgers U., Piscataway, NJ 08854, USA

${ }^{3}$ LeRoy Eyring Center for Solid State Science, Arizona State U., Tempe, AZ 85287, USA

Exploring matter with a $10 \mathrm{meV}$-wide, atom-sized electron probe would allow us to perform many radically new electron energy loss spectroscopy (EELS) experiments, including investigations of atomic vibrations of very small amounts of matter, perhaps down to single atoms. The High Energy Resolution Monochromated EELS STEM (HERMES ${ }^{\mathrm{TM}}$ ) system we have recently developed is now close to being able to reach such performance.

HERMES $^{\mathrm{TM}}$ uses several new design principles [1-3]. The monochromation is carried out at the full primary energy rather than inside the electron gun, with an all-magnetic monochromator (MC) consisting of a main energy-dispersing prism, spectrum-magnifying quadrupoles, auxiliary prisms that bend the beam into an alpha-type trajectory, an adjustable energy-selecting slit equipped with intensity sensing, and aberration-correcting sextupoles and octupoles. The MC optics are dispersing-undispersing, and the energy dispersion of the beam is precisely cancelled at the MC exit. The main prism winding is connected in series with the prism of an all-magnetic EEL spectrometer, which means that the energy spectra produced in these two parts of the total system track each other, and small instabilities in the prism supply current do not change the position of the spectrum on the EELS detector. The high tension (HT) of the microscope is stabilized by sensing the electron current falling on the two halves of the MC slit in a feedback scheme that adjusts the HT such that the energy-dispersed beam remains precisely centered on the slit opening.

Our approach is more complicated than other MC designs - it is roughly equivalent to two parallel EELS systems running back-to-back. However, it provides several key advantages. First, because the beam traverses the MC at the full energy and the beam intensity is reduced by a pre-MC beamdefining aperture, Coulomb interactions between the beam electrons are greatly reduced, allowing high beam currents (e.g., $100 \mathrm{pA}$ at $100 \mathrm{meV}$ energy width) to be reached without broadening the beam unduly. Second, connecting the MC and EELS prisms in series, which is possible precisely because the MC operates on the full-energy beam, results in excellent short- and especially longterm energy stability. Third, full energy electrons are less sensitive to effects such as charging at the slit edges, and this allows the system to achieve very good spatial resolution while monochromating.

Other key components of the total system are an optimized cold field emission gun (CFEG), which provides a coherent current normalized by its energy spread [2] that is about 10x higher than possible with Schottky guns, an aberration corrector that achieves complete correction of geometric aberrations up to $5^{\text {th }}$ order, and a total STEM system designed for the best stability possible with currently available technologies $[4,5]$.

HERMES $^{\mathrm{TM}}$ has been able to reach $12 \mathrm{meV}$ full-width at half-maximum (FWHM) of the EELS zero loss peak (ZLP) in a short-exposure spectrum at a primary energy of $60 \mathrm{keV}$ (Fig. 1), and an energy 
stability better than $20 \mathrm{meV}$ r.m.s. over 100 seconds at $100 \mathrm{keV}$ (Fig. 2). It is routinely able to operate with 30-40 meV energy resolution. When the energy-selecting slit is opened to admit an electron beam about $100 \mathrm{meV}$ wide, the spatial resolution the system can reach is slightly improved compared to running with the MC turned off (and the beam going straight through it), or the MC on and the energy-selecting slit withdrawn from the beam [6]. Fig 3. shows EEL spectra of an $\mathrm{SiO}_{2}$ layer in a MOSFET device acquired with about $40 \mathrm{meV}$ energy resolution at $60 \mathrm{keV}$.

Further improvements in the energy resolution should be possible when the detector part of the column and the EELS (Gatan Enfinium) are provided with similar stability measures as the probeforming part of the column, which is able to keep the sample-level probe stable to around $0.1 \AA$ r.m.s. [7]. We are now working in this direction, and we will report on our progress at the meeting.

[1] O.L. Krivanek et al. (2009), Phil. Trans. Roy. Soc. A367, 3683.

[2] O.L. Krivanek et al. (2013), Microscopy, in print (doi: 10.1093/jmicro/dfs089).

[3] O.L. Krivanek and N. Dellby (2013), US patent \#8373137.

[4] O.L. Krivanek et al. (2008), Ultramicroscopy 108, 179-195.

[5] N. Dellby et al. (2011), EPJAP 54, 33505 (11 pages).

[6] T.C. Lovejoy et al. (2013), these proceedings.

[7] O.L. Krivanek et al. (2010), Microscopy Microanalysis 16 (Suppl. S2), 70-71.
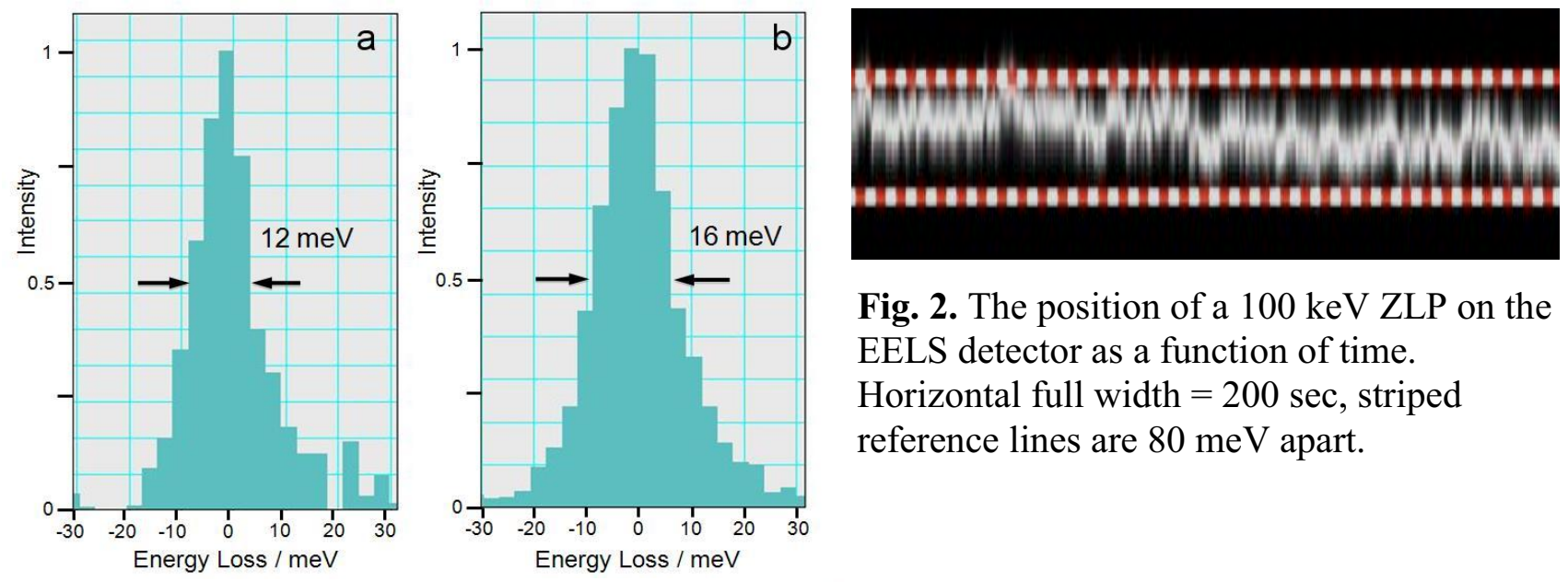

Fig. 2. The position of a $100 \mathrm{keV}$ ZLP on the EELS detector as a function of time. Horizontal full width $=200 \mathrm{sec}$, striped reference lines are $80 \mathrm{meV}$ apart.

Fig. 1. ZLPs acquired at $60 \mathrm{keV}$ in a) $2 \mathrm{~ms}$ and b) $55 \mathrm{~ms}$.
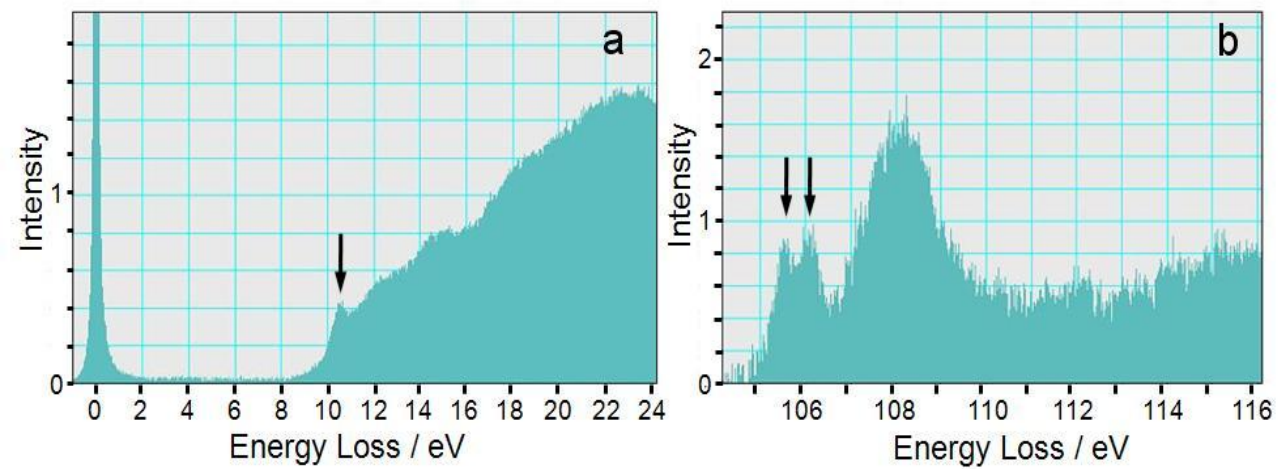

Fig. 3. Si spectra from $\mathrm{SiO}_{2}$ in a MOSFET integrated circuit. a) low loss spectrum acquired in $0.2 \mathrm{~s}$, b) background-subtracted $\mathrm{Si} \mathrm{L}_{2,3}$ edge acquired in $50 \mathrm{~s}$. The ZLP maximum intensity is 302 . Band edge exciton is marked by an arrow in (a), $0.6 \mathrm{eV} \mathrm{Si} \mathrm{L}_{3}-\mathrm{L}_{2}$ threshold peak splitting is marked in (b). 